\title{
Genetic Fuzzy Systems: Status, Critical Considerations and Future Directions
}

\author{
Francisco Herrera \\ Department of Computer Science and Artificial Intelligence, \\ University of Granada, \\ 18071 Granada, Spain \\ herrera@decsai.ugr.es
}

\begin{abstract}
Fuzzy Systems have shown their utility for solving a wide range of problems in different application domains. The use of Genetic Algorithms for designing Fuzzy Systems allows us to introduce the learning and adaptation capabilities. This topic has attracted considerable attention in the Computation Intelligence community.

This short paper briefly reviews the classical models and the most recent trends for Genetic Fuzzy Systems. We pay special attention to a short discussion on some critical considerations of recent developments and to the suggestion of potential research future directions.
\end{abstract}

Keywords: Fuzzy rule based systems, genetic algorithms, genetic fuzzy systems.

\section{Introduction}

Computational Intelligence techniques such as artificial neural networks, fuzzy logic, and genetic algorithms (GAs) are popular research subjects, since they can deal with complex engineering problems which are difficult to solve by classical methods [27].

Hybrid approaches have attracted considerable attention in the Computational Intelligence community. One of the most popular approaches is the hybridization between Fuzzy Logic and GAs leading to genetic fuzzy systems (GFSs) [12]. A GFS is basically a fuzzy system augmented by a learning process based on a GA.

GAs are search algorithms based on natural genetics that provide robust search capabilities in complex spaces, and thereby offer a valid approach to problems requiring efficient and effective search processes [20].

Fuzzy systems are one of the most important areas for the application of the Fuzzy Set Theory. Fuzzy systems have been successfully applied to solve different kinds of problems in various application domains. In order to show that, Table 1 presents a short analysis of the specialized literature on Fuzzy Systems that we can find at the site of "ScienceDirect Elsevier Science Journals" (http://www.sciencedirect.com). We look for the number of papers published during 2003-2005 in fields classified as
Table 1. fuzzy systems applications by subject areas 2003-05 (sept. 2005)

\begin{tabular}{lr}
\hline \hline \multicolumn{1}{c}{ SUBJECT AREAS } & $\begin{array}{c}\text { Number of } \\
\text { publications }\end{array}$ \\
\hline Agricultural and Biological Sciences & 19 \\
Biochemistry, Genetics and Molecular Biology & 2 \\
Chemical Engineering & 10 \\
Chemistry & 4 \\
Earth and Planetary Sciences & 13 \\
Energy & 46 \\
Engineering & 450 \\
Environmental Science & 23 \\
Material Science & 7 \\
Medicine & 5 \\
Neuroscience & 12 \\
Physics and Astronomy & 17 \\
Social Sciences & 12 \\
\hline \hline
\end{tabular}

subject areas by ELSEVIER. They can be found through the query: <fuzzy rule> or <fuzzy system> or < fuzzy control> within "Abstract, title and keywords" for every set of journals associated to a subject area.

On the other hand, in order to show the activity of the GFS area, we present the number of journal papers that have been found in the mentioned ELSEVIER site and the IEEE site "IEEE All-Society Periodicals Package (ASPP)" (http://ieeexplore.ieee.org/Xplore/dynhome.jsp using the "IEEE Periodical" link). Table 2 shows these data classifying them into three periods, 1997-1999, 2000-2002 and 2003-2005. The journal papers can be found through the query: (<fuzzy rule> or <fuzzy control>) and < genetic algorithm $>$ within "Abstract, title and keywords" at the ELSEVIER site and within "Abstract" at the IEEE site.

Table 2. GFSs Publications at the ELSEVIER And IEEE Sites

\begin{tabular}{lrr}
\hline \multirow{2}{*}{ YEAR } & \multicolumn{2}{c}{ Number of publications } \\
& ELSEVIER & IEEE \\
\hline $1997-1999$ & 51 & 20 \\
$2000-2002$ & 57 & 19 \\
$2003-S e p t .2005$ & 55 & 17
\end{tabular}

We only have used the term GAs as evolutionary approach, of course, we would find more publications if we 
include other evolutionary keywords such us: genetic programming (GP), evolutionary algorithms (EAs), ...

We can see a similar number of papers per period, which can give us the idea that it is an active and mature area.

A deep analysis of the literature shows that the most prominent types of GFSs are genetic fuzzy rule based systems (GFRBSs) [12], whose genetic process learns or tunes different components of a fuzzy rule-based system (FRBS). Fig. 1 illustrates graphically this idea. Within GFRBSs it is possible to distinguish between either parameter optimisation or rule generation processes, that is, adaptation and learning.

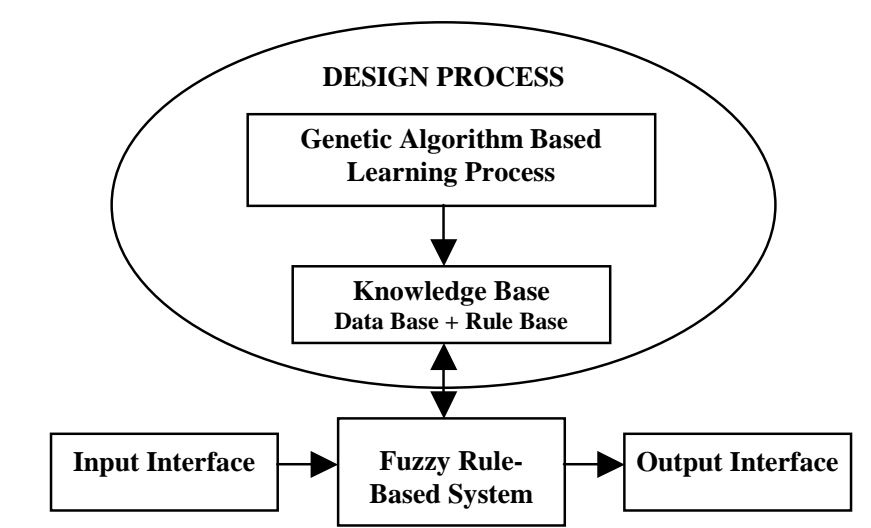

\begin{tabular}{|l|l|l|}
\hline Environment & Computation with Fuzzy Rule-Based Systems & Environment \\
\hline
\end{tabular}

Figure 1. Genetic fuzzy systems.

The paper starts by briefly reviewing FRBSs and GAs in Section II. Next we present the current status of GFRBSs in Section III. Section IV introduces a short discussion on some critical considerations on recent publications in the area. Section $\mathrm{V}$ presents some suggestions of potential future research directions. Finally, some concluding remarks are pointed out in Section VI.

\section{Preliminaries: FRBSs and GAs}

\section{A. Fuzzy Rule Based Systems}

The essential part of an FRBS is a set of IF-THEN linguistic rules, whose antecedents and consequents are composed of fuzzy statements, related by the dual concepts of fuzzy implication and the compositional rule of inference.

An FRBS is composed by a knowledge base $(\mathrm{KB})$, that includes the information given by the expert in the form of linguistic fuzzy rules; a Fuzzification Interface, which has the effect of transforming crisp data into fuzzy sets; an Inference System, that uses them together with the KB to make inference by means of a reasoning method; and a Defuzzification Interface, that translates the fuzzy rule action thus obtained to a real action using a defuzzification method. The generic structure of an FRBS is shown in Fig. 2.
The KB encodes the expert knowledge by means of a set of IF-THEN rules, which are a conditional statement with the form:

\section{IF a set of conditions are satisfied \\ THEN a set of consequents can be inferred}

in which the antecedent is a condition in its application domain, the consequent is an output action to be applied in the system and both antecedent and consequent are associated with fuzzy concepts, that is, linguistic terms (notion of fuzzy rule).

The KB is comprised of two components:

- A data base (DB), containing the linguistic term sets considered in the linguistic rules and the membership functions defining the semantics of the linguistic labels. Each linguistic variable involved in the problem will have associated a fuzzy partition of its domain representing the fuzzy set associated with each of its linguistic terms.

- A rule base (RB), comprised of a collection of linguistic rules that are joined by a rule connective ("also" operator). In other words, multiple rules can fire simultaneously for the same input.

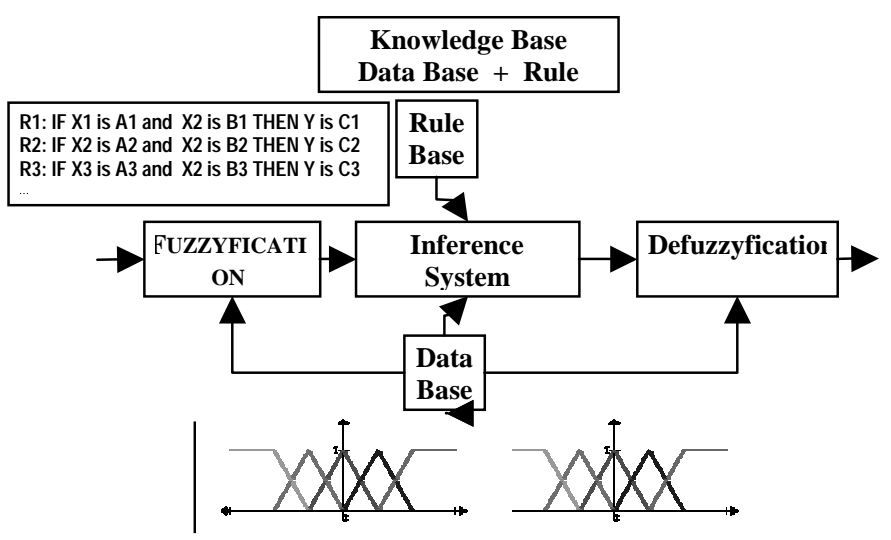

Figure 2. Generic structure of a fuzzy rule based system.

For more information about fuzzy systems the following books may be consulted: [12 ] and [ 40].

\section{B. Genetic Algorithms}

GAs are general purpose search algorithms which use principles inspired by natural genetics to evolve solutions to problems [20]. The basic idea is to maintain a population of chromosomes (representing candidate solutions to the concrete problem being solved) that evolves over time through a process of competition and controlled variation.

A GA starts with a population of randomly generated chromosomes, and advances towards better chromosomes by applying genetic operators modelled on the genetic processes occurring in nature. The population undergoes evolution in a form of natural selection. During successive 
iterations, called generations, chromosomes in the population are rated for their adaptation as solutions, and on the basis of these evaluations, a new population of chromosomes is formed using a selection mechanism and specific genetic operators such as crossover and mutation. An evaluation or fitness function must be devised for each problem to be solved. Given a particular chromosome, the fitness function returns a single numerical value, which is supposed to be proportional to the utility or adaptation of the solution represented by that chromosome.

Although there are many possible variants of the basic GA, the fundamental underlying mechanism consists of three operations: evaluation of individual fitness, formation of a gene pool (intermediate population) through selection mechanism, and recombination through crossover and mutation operators. The generic structure of a GA is illustrated in Fig. 3.

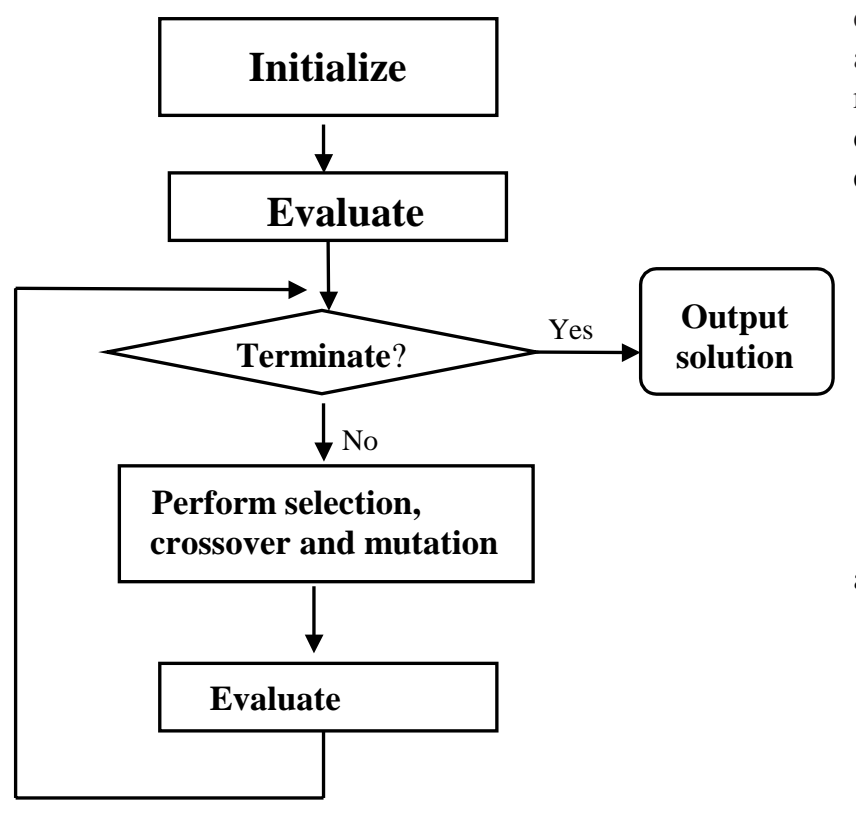

Figure 3. Genetic Algorithm Flowchart

The application of a GA to solve a problem must determine:

- A genetic representation (called genotype) of the solutions of the problem (called phenotype).

- A way to create an initial population of solutions.

- An evaluation function (called fitness function) which provides a quality value to each chromosome.

- Operators which modify the genetic composition of the descendants during reproduction.

- Values for the parameters used (population size, probabilities of application of genetic operators, etc.).

Bäck et al. in [4] gives a complete description of GAs as well as other examples of EAs.
Although GAs were not specifically designed for learning, but rather as global search algorithms, they offer a set of advantages for machine learning. Many methodologies for machine learning are based on a search of a good model inside a space of models, such as the space of rule sets. These methodologies may model the learning problem as a search problem or as an underlying optimization. GAs perform the search in the space of models by means of the codification of the model in a chromosome. In this sense, they are very flexible because the same GA can be used with different representations.

Genetic learning processes cover different levels of complexity according to the structural changes produced by the algorithm, from the simplest case of parameter optimization to the highest level of complexity of learning the rule set of a rule-based system. Genetic learning processes designed for parameter optimization usually fit to the description given in previous paragraphs, but when considering the task of learning rules in a rule based system, a wider range of possibilities is open. When considering a rule based system and focusing on learning rules, the different genetic methods follow two approaches in order to encode rules within a population of individuals [12]:

- The "Chromosome = Set of rules", also called the Pittsburgh approach, in which each individual represents a rule set [14].

The "Chromosome = Rule" approach, in which each individual codifies a single rule, and the whole rule set is provided by combining several individuals in the population.

In turn, within the "Chromosome = Rule" approach, there are three generic proposals:

- The Michigan approach, in which each individual codifies a single rule. These kinds of systems are usually called learning classifier systems. They are rulebased, message-passing systems that employ reinforcement learning and the GA to learn rules that guide their performance in a given environment [28].

- The IRL (Iterative Rule Learning) approach, in which each chromosome represents a rule, but the solution is the best individual obtained and the global solution is formed by the best individuals obtained when the algorithm is run multiple times. MOGUL [10,23] and SLAVE [21] are proposals that follow this approach.

- The "cooperative-competitive" approach, in which the complete population or a subset of it codifies the rule base. COGIN [22], REGAL [19] and LOGENPRO [42] are examples with this kind of representation.

\section{Genetic Fuzzy Systems: Status}

The central aspect on the use of a GA for automatic learning of an FRBS is that the KB design process can be analyzed as an optimization problem.

From the optimization point of view, to find an appropriate $\mathrm{KB}$ is equivalent to coding it as a parameter structure and 
then to find the parameter values that give us the optimum for a fitness function. The KB parameters provide the search space that is transformed according to a genetic representation. Therefore, the first step in designing a GFRBS is to decide which parts of the KB are subject to optimization by the GA.

In the following, we shortly describe the classical GFRBSs approaches together with the new trends that appear exploring new directions to apply GAs to FRBSs design.

\section{A. Classical GFRBSs Approaches}

Two problems arise when generating the KB [12]:

- The DB learning, that comprises the specification of the universes of discourse and the number of labels for each linguistic variable, as well as the fuzzy membership functions associated to each label.

- The RB learning, involving the determination of the number of rules and of the composition of each one of them (i.e., of the specific labels associated to each linguistic variable).

In the following, we shortly analyze the four kinds of approaches followed in the design of a GFRBSs for learning the KB:

1) Genetic learning of the $R B$. Most of the approaches proposed to automatically learn the $\mathrm{KB}$ from numerical information have focused on the RB learning, using a predefined DB. The usual way to define this DB involves choosing a number of linguistic terms for each linguistic variable (an odd number between 3 and 9, which is usually the same for all the variables) and setting the values of the system parameters by an uniform distribution of the linguistic terms into the variable universe of discourse. Fig. 4(a) graphically shows this type of KB learning.
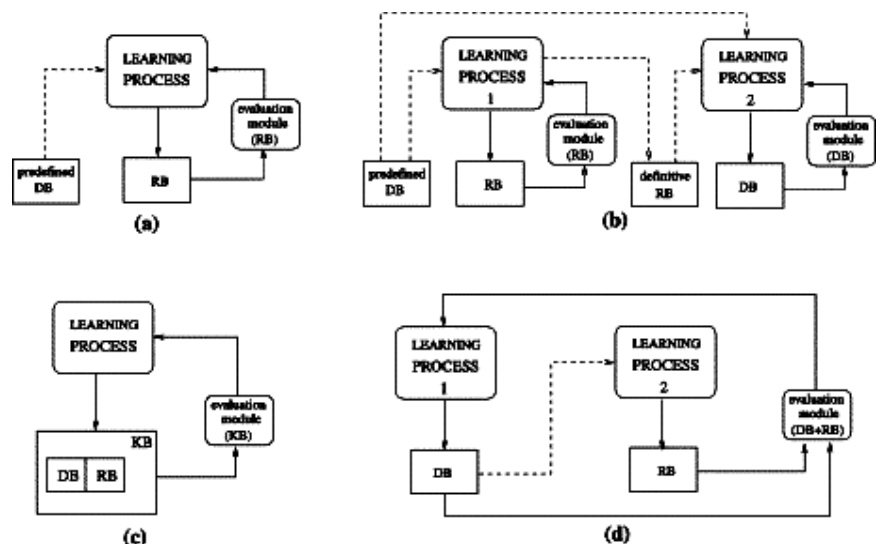

Figure 4. Graphical representation of different KB learning approaches
2) Genetic tuning. With the aim of making the FRBS perform better, some approaches try to improve the preliminary $\mathrm{DB}$ definition once the RB has been derived. To do so, a tuning process considering the whole $\mathrm{KB}$ obtained (the preliminary $\mathrm{DB}$ and the derived $\mathrm{RB}$ ) is used a posteriori to adjust the membership function parameters. Nevertheless, the tuning process only adjusts the shapes of the membership functions and not the number of linguistic terms in each fuzzy partition, which remains fixed from the beginning of the design process. It can be also named as learning of the RB and, a posteriori, the DB. A graphical representation of this kind of learning is showed in Fig. 4(b).

3) Genetic learning of the $K B$. Other approaches try to learn the two components of the $\mathrm{KB}$ simultaneously. This kind of learning is depicted in Fig. 4(c). Working in this way, they have the possibility of generating better definitions but they deal with a larger search space that makes the learning process more difficult and slow.

4) Genetic learning of the DB a priori. Finally, there is another way to generate the whole $\mathrm{KB}$ that considers two different processes to derive both components, DB and RB. A DB generation process wraps an RB learning one working as follows: each time a DB has been obtained by the DB definition process, the RB generation method is used to derive the rules, and some type of error measure is used to validate the whole $\mathrm{KB}$ obtained. We should note that this operation mode involves a partitioning of the $\mathrm{KB}$ learning problem. Whilst the learning processes belonging to the previous family (Fig.4(c)) look for solutions in a complex global search space $(\mathrm{DB}+\mathrm{RB})$, the processes belonging to the current group are composed of two different (and independent) learning processes looking for solutions in two simpler search spaces (DB and RB ones) to obtain complete solutions. This type of $\mathrm{KB}$ learning is represented in Fig. 4(d).

\section{B. New Trends in GFRBSs}

In addition to the classical systems addressed in previous subsection, new directions to apply genetic (evolutionary) techniques to FRBSs have been explored. A short summary of them is shown in the following:

- Designing FRBS with genetic programming.

- Genetic selection of fuzzy rule sets

- Genetic feature selection

- Maintaining interpretability via multi-objective genetic processes

- Genetic-based learning approaches considering different model structures: double-consequent fuzzy rules, weighted fuzzy rules, hierarchical KBs,...

- Genetic-based learning approaches with sophisticated GAs.

We must remark that the coding flexibility of the GAs allow us to represent different kind of structures, such as weights, features together with rules parameters, etc., allowing to code multiple models of knowledge 
representation. This provides a wide variety of approaches where it is necessary to design specific genetic components for evolving a specific representation (chromosome).

A short description on these non classical explored approaches together with a complete set of references can be found in [11].

\section{GFSs: Critical Considerations On Recent Advances}

The question that I proceed to discuss in this section is related to some common features of an important percentage of recent publications in the topic.

In the last years, we can find a lot of publications that present a "novel" proposal for evolving a KB or a specific component (RB or DB). When we read the abstract we feel a great interest to read the specific aspects of the proposal, and the most important, to see the results and the comparative analysis against the well known approaches in the GFS literature in particular, or fuzzy systems literature in general.

What are the critical points for a big number of recent published papers?

In the following six points I discuss the critical issues that we can find in a big number of recent publications proposing new GFS algorithms. These critical points can be extended to other fuzzy rule learning approaches.

1) Repeating approaches. We find proposals that are very similar to other well known ones. Authors do not present a state of the art on the topic. They do not include in their contribution a comparative analysis with the existing ones. Authors do not describe the real advantages/novelties of the proposal against those with similar features/components.

Authors and referees must take in consideration the state of the art in the writing and referee processes, respectively. It is necessary to advance to real novel approaches but not to present the same models over and over.

2) Simple GAs. In an important number of contributions we find a description of the most simple GAs (see code in [20]), with a classical parameter coding (binary alphabet) and simple components.

There exist a wide literature on GAs in particular, and EAs in general with important approaches that introduce important advances. Some examples are, the real coding for continuous variables, tournament selection against the roulette one, different parent replacement strategies, adaptive components, etc. On the other hand, there are specific kinds of GAs for different tasks, such as, niching GAs for multimodal functions, hybrid combinations of GAs and local search (called memetic algorithms), etc.

Authors must really know GAs components and models before applying a simple GA, and choose an adequate algorithm, if necessary, for getting a good evolutionary model.
3) The use of novel EAs. Recently it is usual to find evolutionary learning proposals that use a classical genetic representation for a KB and use a novel EA (such as particle swarm, cultural genetic algorithms, etc.) but authors do not compare them with the classical GFS that we can find in the literature. Researchers claim that the novel EA provides very good results, but do not offer any justification for their use.

The use of a novel EA must be justified from whatever meaningful point of view: efficiency, efficacy/precision, interpretability, scalability, etc.

4) Benchmark problems. When we read the experimental analysis we usually find a different set of benchmarks problems in every paper. In particular, we can find specific applications for learning from data without any possibility for getting the data set, therefore it is impossible to reproduce the same experimental study. In the same way, it is not possible to reproduce some algorithms due to the lack of the parameters values used by the authors in the experimental study.

It is necessary to manage adequate and unified sets of benchmark problems for learning from data, providing all the necessary information for reproducing the experimental study.

We are working in this sense and we are preparing a benchmark site for problems and data sets for unifying the experimentation. The set is called KEELlib and it can be found at the our project site: http://sci2s.ugr.es/keel.

5) Lack of experimental statistical analysis. Another critical point is related to the comparative study. Contrary to other machine learning approaches, currently there is not a systematic evaluation methodology for GFSs.

Experimental results reported in the machine learning literature often use statistical tests of significance to support the claim that a new learning algorithm generalizes better. In fact, the performance analysis of learning algorithms has always centred the attention of investigators in the machine learning area, and different comparison proposals have been developed (cross validation, uses of $5 \times 2 \mathrm{cv}$, leave one out, etc.) in terms of their type I and type II errors, both on synthetic datasets, and standard benchmarks of machine learning [15,32]. This is an important problem that presents recent and interesting studies. We would like to point at two recent studies: a) the problem of estimating the variance of the cross-validation estimator of the generalization error, paying special attention to the variability introduced by the selection of a particular training set [33], and b) a method for deriving variance estimators of the cross validation estimator of the generalization error, using the method of moment approximation [34].

The use of statistical analysis tools is a necessity in the analysis of GFS models as it is in classical machine learning. It is a perentorious necessity their use for analysing the generalization performance of GFSs when the algorithm aims at reaching a maximum accuracy.

6) Interpretability quality. Of course, there exist another important feature for measuring the FRBS quality, the model interpretability. Interpretability is considered to be the main advantage of fuzzy systems over alternatives like 
neural networks, statistical models, etc. As authors claim in [35], interpretability means that humans beings are able to understand the fuzzy system's behaviour by inspecting the RB. Fuzzy systems constructed from expert knowledge -the traditional approach- usually are well understandable.

In the recent years, research has started to focus on the trade-off between interpretability and accuracy $[7,8]$. Analysis of the model interpretability and comprehensibility is always convenient, and it is a necessity when accuracy is not a model feature.

A GFS model providing an FRBS with low interpretability and low accuracy is not a valuable contribution. Therefore, both aspects would be analyzed in depth by authors and reviewers.

I consider these six aspects as critical points to advance towards the right issues, that is, to concentrate more on the strengths and distinctive features of the GFSs, providing an useful advance in the fuzzy systems theory.

\section{GFSs: Future Directions}

At the present, I consider the field of GFSs as a mature area, that needs to advance towards new questions and problems. In what follows, I enumerate four research directions that are worth to continuous the exploration, or initiate in some aspects.

1) Trade-off interpretability versus precision. The use of Multi-objective Genetic Algorithms (MOGAs). Obtaining high degrees of interpretability and accuracy is a contradictory purpose, and, in practice, one of the two properties prevails over the other. Nevertheless, a new tendency in the fuzzy modeling scientific community that looks for a good balance between interpretability and accuracy is increasing in importance. The improvement of the interpretability of rule based systems is a central issue in recent research, where not only the accuracy is receiving attention but also the compaction and the interpretability of the obtained rules $[29,35]$.

Whereas the definition of accuracy in a certain application is straightforward, the definition of interpretability is rather problematic. Most researchers would agree in interpretability involving aspects as: the number of rules is enough to be comprehensible, rules premises should be easy in structure and contain only a few input variables, linguistic terrns should be intuitively comprehensible.

We need to propose new interpretability metrics that consider not only the number of rules but other aspects as the number of labels of a rule, the shape of the membership functions, etc. with a better understanding and formalization of the notions of "interpretability", "comprehensibility" or "simplicity". More research is need in form of evaluation metrics for giving an interpretability measure associated to an FRBSs allowing us to compare different FRBSs for a problem from the interpretability point of view. We claim on the interpretability but without a metric for measuring it (see $[2,3]$ as example of this situation).
MOGAs is an important research line within GAs due to population-based algorithms are capable of capturing a set of non-dominated solutions in a single run of the algorithm. The use of MOGAs $[9,13]$ allows us to include both the model accuracy and metrics of interpretability and compactness $[25,39,41]$.

In multiobjective GFSs is desirable to design genetic learning algorithms in which the learning mechanism itself finds an appropriate balance between interpretability and accuracy. These models should result in small and simple rule sets, contributing significantly to the user knowledge because of its interpretability. Currently it is an active and interesting research line that also will provide useful results in near future.

2) FRBSs for high dimensional problems. It is usual to find big databases, i.e., with high number of features and/or instances. Regarding to the interpretability of linguistic FRBSs, the difficult comes from the exponential growth of the fuzzy rule search space with the increase in the number of features/instances considered. Usually human users do not want to check hundreds of fuzzy rules, the number of fuzzy rules is closely related to the interpretability of FRBSs. On the other hand, the rule length is also closely related to the interpretability of FRBSs.

This problem can be tackled by different ways: a) compacting and reducing the rule set as a post-processing approach, b) carrying out a feature selection process, that determines the most relevant variables before or during the inductive learning process of the FRBS, and c) removing irrelevant training instances prior to FRBS learning. The two first approaches have been tried in the specialized literature (see previous New Trends subsection), but the last one, until our knowledge in the topic, has not be used for learning FRBS. It has been used for extracting decision trees $[6,38]$.

Feature and instance selection (data reduction techniques) allows smaller training sets, which may yield to more accurate and more compact models. And in both cases, GAs are used frequently, because the selection problem may be defined as the problem of searching the optimal subset of features/instances.

The inclusion of genetic data reduction processes inside of the GFS model is a research direction that allows us to advance in the extraction of FRBS with an appropriate balance between interpretability and accuracy in high dimensional problems.

I also would like to point at the use of GP for learning compact FRBSs. The GP is an extension to the inspiration of $\mathrm{GA}$, where the main problem of GA concerning the fixed problem definition is avoided by using variable-length trees instead of fixed-sized individuals [30]. The definition of context-free grammars for rule construction has been revealed of special utility for this purpose [42]. The use of GP in a GFS model can lead us to obtaining a reduced fuzzy rule set, with few antecedents conditions per rule and highgeneralization capability, getting FRBSs with high interpretability for high-dimensionality problems. 
Another problem when we deal with high dimensional problems is the analysis of the algorithm scalability on big databases, emphasizing the training time and the convergence towards compact and interpretable models. The balance between problem size and algorithm scalability is another important aspect for GFSs that worth to be study in depth. At this point, we must remark the existence of efficient parallel GAs [1] as a kind of GAs that would be evaluated for designing GFS for big databases putting special emphasis on aspects of scalability and efficiency.

3) GFSs in Data Mining (DM) and Knowledge Discovery (KD). Association rules, subgroup discovery and patterns identification. Fayyad et al. [17] defined KD as the nontrivial process of identifying valid, novel, potentially useful, and ultimately understandable patterns in data. KD may not be viewed as a synonymous with DM, but they are intimately related. $\mathrm{KD}$ is a wide ranging process which covers distinct stages: the comprehension of the problem, the comprehension of the data, pre-processing (or preparation) of the data, DM and post-processing (assessment and interpretation of the models). The DM stage is responsible for automatic KD of a high level and from information obtained from real data. Some of the important problems that $\mathrm{DM}$ and $\mathrm{KD}$ deal with are: rule extraction, identification of associations, feature analysis, linguistic summarization, clustering, classifier design, and novelty/anomaly detection.

The interpretability is crucial in the field of $\mathrm{DM} / \mathrm{KD}$ where knowledge should be extracted from data bases and represented in a comprehensible form, or for decision support systems where the reasoning process should be transparent to the user. In fact, the use of linguistic variables and linguistic terms in a discovered process has been explored by different authors.

As it was claimed by Dubois et al. in [16], the use of fuzzy sets to describe association between data extends the types of relationships that may be represented, facilitates the interpretation of rules in linguistic terms, und avoids unnatural boundaries in the partitioning of the attribute domains.

I would like to pay attention on a subdivision of descriptive induction algorithms which has recently received attention from researchers, it is called subgroup discovery. It is a form of supervised inductive learning of subgroup descriptions in which, given a set of data and having a property of interest to the user, attempts to locate subgroups which are statistically "most interesting" for the user.

Subgroup discovery has the objective of discovery interesting properties of subgroups obtaining simple rules (i.e. with an understandable structure and with few variables), highly significant and with high support (i.e. covering many of the instances of the target class). The concept was initially formulated by Klösgen in his rule learning algorithm EXPLORA [26] and by Wrobel in the algorithm MIDOS [43]. Both use a rule-extraction model based on decision trees, in order to obtain the best subgroups among the population. In order to evaluate the subgroups, evaluation measurements are defined which determine the interest of an expression through a combination of unusualness and size. MIDOS tackles, within this same approach, the problem of discovery in multi-relational databases. A recent study can be found in [31].

Linguistic variables with linguistic terms can contribute in a substantial way to advance in the design of association rules and subgroup discovery, in particular, and the analysis of data to establish relationships and identify patterns, in general [24]. On the other hand, GAs in particular, and EAs in general, are widely used for evolving rules extraction and patterns association in DM/KD [18]. The conjunction in the GFS field provide novel useful tools for patterns analysis and for extracting new kinds of useful information with a main advantage over other techniques, its interpretability in terms of fuzzy if-then rules.

4) Learning genetic models based on vague data. The experimental designs of GFSs learning from data observed in an imprecise way are not being actively studied by researchers. However, according to the point of view of fuzzy statistics, the primary use of fuzzy sets in classification and modelling problems is for the treatment of vague data [5]. Using vague data to train and test GFSs we could analyze the performance of these classifiers on the type of problems for which fuzzy systems are expected to be superior. Preliminary results in this area involve the proposals of different formalizations for the definition of fuzzy classifiers, based on the relationships between random sets and fuzzy sets [36,37], and the study of fitness functions (with fuzzy values) that should be defined in the context of GFSs [37].

This is a novel area that is worth to explore in the near future, that can provide interesting and promising results.

This is not a complete list of potential research directions for GFS, of course, but it is a set of personal reflections on some potentials research lines for investigation.

\section{Conclusions}

The hybridization between fuzzy systems and GAs in GFSs became an important research area during the last decade. At the present, it is a mature research area, where researchers need to reflect in order to advance towards strengths and distinctive features of the GFSs, providing useful advance in the fuzzy systems theory.

Finally, I would like to finish with two considerations on this paper:

- It does not try to be a directory to guide the reader to a set of references, beginners in the topic can get two important list of references in [11,12].

- It tries to introduce shortly the topic, to call the attention on critical points that need to be tackled for researchers working with GFSs and fuzzy knowledge extraction algorithms, and to shortly discuss some research lines that I consider as "burning issues". 


\section{References}

[1] E. Alba, M. Tomassini, "Parallelism and evolutionary algorithms," IEEE Transactions on Evolutionary Computation, vol. 6, pp. 443-462, 2002.

[2] R. Alcalá, J. Alcalá-Fdez., F. Herrera, J. Otero, "Genetic Learning of the Knowledge Base of a Fuzzy System by Using the Linguistic 2-Tuples Representation," in Proceedings of the 14th IEEE International Conference on Fuzzy Systems (FUZZIEEE 2005), Reno, Nevada (USA), pp. 797-802, IEEE Press, 2005.

[3] R. Alcalá, F. Herrera, "Genetic Tuning on Fuzzy Systems Based on the Linguistic 2-Tuples Representation," in Proc. of the $13^{\text {th }}$ IEEE International Conference on Fuzzy Systems (FUZZIEEE 2004), Budapest (Hungary), pp. 233-238, IEEE Press, 2004

[4] T. Bäck, D. Fogel, Z. Michalewicz, Handbook of Evolutionary Computation. Oxford, UK: Oxford University Press, 1997.

[5] C. Bertoluzza, M. A. Gil, D.A. Ralescu (Eds.). Statistical Modeling, Analysis and Management of Fuzzy Data. Springer-Verlag., 2003.

[6] J.R. Cano, F. Herrera, M. Lozano, "On the combination of evolutionary algorithms and stratified strategies for training set selection in data mining," Applied Soft Computing, 2006, in press.

[7] J. Casillas, O. Cordón, F. Herrera, L. Magdalena (Eds.), Accuracy Improvements in Linguistic Fuzzy Modeling. Springer-Verlag, 2003.

[8] J. Casillas, O. Cordón, F. Herrera, L. Magdalena (Eds.), Interpretability Issues in Fuzzy Modeling. Springer-Verlag, 2003.

[9] C.A. Coello, D.A. Van Veldhuizen, G.B. Lamont, Evolutionary Algorithms for Solving Multi-Objective Problems. Kluwer Academic Publishers, 2002.

[10] O. Cordón, M.J. del Jesús, F. Herrera, M. Lozano, "MOGUL: A Methodology to Obtain Genetic fuzzy rule-based systems Under the iterative rule Learning approach," International Journal of Intelligent Systems, vol. 14, pp. 1123-1153, 1999.

[11] O. Cordón, F. Gomide, F. Herrera, F. Hoffmann, L. Magdalena, "Ten years of genetic fuzzy systems: Current framework and new trends," Fuzzy Sets and Systems, vol. 41, pp. 5-31, 2004.

[12] O. Cordón, F. Herrera, F. Hoffmann, L. Magdalena, Genetic Fuzzy Systems. Evolutionary Tuning and Learning of Fuzzy Knowledge Bases. World Scientific, 2001.

[13] K. Deb, Multi-Objective Optimization using Evolutionary Algorithms. John Wiley \& Sons, 2001

[14] K.A. De Jong, W.M. Spears, D.F. Gordon, "Using genetic algorithms for concept learning," Machine Learning, vol. 13, pp. 161-188, 1993.

[15]T. Diettereich, "Approximate statistical tests for comparing supervised classification learning algorithms," Neural Computation, vol. 10, pp. 18951924, 1998.

[16]D. Dubois, H. Prade, T. Sudamp, "On the representation, measurement, and discovery of fuzzy associations," IEEE Trans. on Fuzzy Systems, vol. 13, pp. 250-262, 2005.

[17] U. Fayyad, G. Piatesky-Shapiro, P. Smyth, "From data mining from knowledge discovery in databases", in U.M. Fayyad, G. Piatetsky-Shapiro, P. Smyth, R. Uthurusamy (Eds.), Advances in Knowledge Discovery \& Data Mining, AAAI/MIT, pp 1-34, 1996.

[18] A.A. Freitas, Data Mining and Knowledge Discovery with Evolutionary Algorithms. Springer-Verlag, 2002.

[19]A. Giordana, F. Neri, "Search-intensive concept induction". Evolutionary Computation, vol. 3, pp. 375416, 1995.

[20] D. E. Goldberg, Genetic Algorithms in Search, Optimization, and Machine Learning. AddisonWesley, 1989.

[21]A. González, R. Pérez, "SLAVE: A genetic learning system based on an iterative approach," IEEE Transactions on Fuzzy Systems, vol. 27, 176-191, 1999.

[22] D.P. Greene, S.F. Smith, "Competition-based induction of decision models from examples," Machine Learning, vol. 3, pp. 229-257, 1993.

[23]F. Herrera, M. Lozano, J.L. Verdegay, “A Learning Process for Fuzzy Control Rules using Genetic Algorithms," Fuzzy Sets and Systems, vol. 100, 143$151,1998$.

[24] E. Hüllermeier, "Fuzzy methods in machine learning and data mining: Status and prospects," Fuzzy Sets and Systems, vol. 156, pp. 387-406, 2005.

[25] H. Ishibuchi, T. Yamamoto, "Fuzzy rule selection by multi-objective genetic local search algorithms and rule evaluation measures in data mining," Fuzzy Sets and Systems 141, pp. 59-88., 2004.

[26] W. Klösgen, "Explora: a multipattern and multistrategy discovery assistant", in U.M. Fayyad, G. PiatetskyShapiro, P. Smyth, and R. Uthurusamy (Eds.), Advances in Knowledge Discovery and Data Mining, MIT Press, pp. 249-271, 1996.

[27] A. Konar, Computational Intelligence: Principles, Techniques and Applications. Springer-Verlag, 2005.

[28] T. Kovacs, Strength or Accuracy: Credit Assignment in Learning Classifier Systems. Springer-Verlag, 2004.

[29] Kweku-Muata, Osey-Bryson, "Evaluation of decision trees: a multicriteria approach," Computers and Operations Research, vol. 31, pp. 1933-1945, 2004.

[30]J.R. Koza, Genetic Programming - on the Programming of Ccomputers by Means of Natural Selection. Cambridge MA, USA: The MIT Press. 1992.

[31]N. Lavrac, B. Cestnik, D. Gamberger, P. Flach, "Decision support through subgroup discovery: three case studies and the lessons learned," Machine Learning, vol. 57, pp. 115-143, 2004.

[32] J.W. Lee, J.B. Lee, M. Park, S.H. Song, “An extensive comparison of recent classification tools applied to 
microarray data," Computational Statistics \& Data Analysis, vol. 48, pp. 869-885, 2005.

[33]C. Nadeau, "Inference for the generalization error," Machine Learning, vol. 52, pp. 239-281, 2003.

[34]M. Markatou, H. Tian, S. Biswas, G.Hripcsak, "Analysis of variance of cross-validation estimators of the generalization error," Journal of Machine Learning Research, vol. 6, pp. 1127-1168, 2005.

[35] R.Mikut, J. Jäkel, L. Gröll, "Interpretability issues in data-based learning of fuzzy systems," Fuzzy Sets and Systems, vol. 150, pp. 179-197, 2005.

[36] L. Sánchez, J. Casillas, O. Cordón, M.J. del Jesus, "Some relationships between fuzzy and random classifiers and models," Int. J. of Approximate Reasoning, vol. 29, pp. 175-213, 2001.

[37]L. Sánchez, I. Couso, "Advocating the use of imprecisely observed data in genetic fuzzy systems," in Proc. of the First Int. Workshop on Genetic Fuzzy Systems (GFS 2005), Granada. Spain., pp. 124-129, March 2005.

[38] M. Sebban, R. Nock, J. H. Cahuchat, R. Rakotomalala. "Impact of learning set quality and size on decision tree performance," Int. J. of Computers, Syst. and Signals, vol. 1, pp. 85-105, 2000.

[39] C. Setzkorn, R.C. Paton, "On the use of multi-objective evolutionary algorithms for the induction of fuzzy classification rule systems," BioSystems, vol. 81, pp. 101-112, 2005.

[40] R.R Yager, D.P. Filev, Essentials of Fuzzy Modeling and Control, John Wiley \& Sons, 1994.

[41] H. Wang, S. Kwong, Y. Jin, W. Wei, K.F. Man. "Multiobjective hierarchical genetic algorithm for interpretable fuzzy rule-based knowledge extraction," Fuzzy Sets and Systems, vol. 149, pp. 149-186, 2005.

[42] M.L. Wong, K.S. Leung, Data Mining using Grammar Based Genetic Programming and Applications. Kluwer Academics Publishers, 2000.

[43]S. Wrobel, "An algorithm for multi-relational discovery of subgroups", in Proceedings of the First European Symposium on Principles of Data Mining and Knowledge Discovery (PKDD), Berlin, pp. 78-87, 1997.

\section{Author Biographies}

Francisco Herrera received the M.Sc. degree in Mathematics in 1988 and the Ph.D. degree in Mathematics in 1991, both from the University of Granada, Spain.

He is currently a Professor in the Department of Computer Science and Artificial Intelligence at the University of Granada. He has published over 100 papers in international journals and he is coauthor of the book "Genetic Fuzzy Systems: Evolutionary Tuning and Learning of Fuzzy Knowledge Bases" (World Scientific, 2001). As edited activities, he has co-edited three international books and co-edited 15 special issues in international journals on different Soft Computing topics, such as, "Preference Modelling", "Computing with Words", "Genetic Algorithms" and "Genetic Fuzzy Systems". He currently serves on the editorial boards of the Journals: Soft Computing, Mathware and Soft Computing, International Journal of Hybrid Intelligent Systems, and International Journal of Computational Intelligence Research. His current research interests include computing with words, preference modelling, data mining and knowledge discovery, data reduction, fuzzy rule-based systems, genetic algorithms, and genetic fuzzy systems. 